\title{
Real-Time Capabilities of a Digital Analyzer for Mixed-Field Assay using Scintillation Detectors
}

\author{
Michael D. Aspinall, Member, IEEE, Malcolm J. Joyce, Member, IEEE, Anthony Lavietes, Senior Member, IEEE, \\ Romano Plenteda, Frank D. Cave, Helen M. O. Parker, Ashley Jones and Vytautas Astromskas
}

\begin{abstract}
Scintillation detectors offer a single-step detection method for fast neutrons and necessitate real-time acquisition, whereas this is redundant in two-stage thermal detection systems using helium-3 and lithium-6, where the fast neutrons need to be thermalized prior to detection. The relative affordability of scintillation detectors and the associated fast digital acquisition systems have enabled entirely new measurement setups that can consist of sizeable detector arrays. These detectors in most cases rely on photo-multiplier tubes which have significant tolerances and result in variations in detector response functions. The detector tolerances and other environmental instabilities must be accounted for in measurements that depend on matched detector performance. This paper presents recent advances made to a high-speed FPGA-based digitizer. The technology described offers a complete solution for fast-neutron scintillation detectors by integrating multichannel high-speed data acquisition technology with dedicated detector high-voltage supplies. This configuration has significant advantages for large detector arrays that require uniform detector responses. We report on bespoke control software and firmware techniques that exploit realtime functionality to reduce setup and acquisition time, increase repeatability and reduce statistical uncertainties.
\end{abstract}

Index Terms-Real time systems, Neutrons, Neutron spectroscopy, Digital signal processing, Coincidence techniques, Data acquisition systems, Detector instrumentation, Neutron imaging, Scintillation detectors

\section{INTRODUCTION}

$\mathbf{T}$ YPICAL fast neutron detectors are liquid scintillators, helium-4 gas detectors [1] and plastic detectors. Fast neutron detectors differentiate themselves from one another by their ability to discriminate neutrons and gamma rays based on pulse shape and their sensitivity.

Organic plastic and liquid scintillators are often used for detection of fast neutrons because of their fast response, sensitivity to fast neutrons and modest cost [2]. Coincidence counting applications benefit from fast response detectors particularly where the ratio of real to accidental coincidences can have a significant impact on the statistical precision of the measurement. The coincidence window for assay applications that utilize scintillators with response times of a few nanoseconds is typically governed by the dynamic range of the neutron flight time (tens of nanoseconds) and depends on the distance from the source to the detector, neutron energy, and cross section of the material to traverse. Conversely, coincidence counting systems that thermalize fast neutrons prior

Manuscript received June 28, 2016; revised December 3, 2016

M. D. Aspinall is with the Biomedical Engineering Department, Aston University, Birmingham, UK. He is the Systems Engineer behind the development of the Hybrid Instruments Ltd. Mixed-Field Analyzer technology (e-mail: m.aspinall@aston.ac.uk or m.aspinall@hybridinstruments.com). to detection (e.g. helium-3 based detection) are dominated by the dynamic range of the time (tens of microseconds) needed to moderate the neutrons [2]. Moreover, the thermalization of fast-neutrons scrambles information on the original energy, direction of travel, and the time of emission. The detection of fast neutrons that retain this information is the prerequisite for many new and interesting applications relating to neutron coincidence, time-of-flight (TOF) and spatial analysis [3].

The high gamma sensitivity of organic scintillators is a major disadvantage in non-destructive assay applications because most nuclear material emits more than 10 times as many gamma rays as neutrons. The high gamma detection probability can overwhelm the detector or processing electronics and dominate the bandwidth making it difficult to obtain sufficient neutron data. This, together with inadequate distinction of event types through pulse-height spectra analysis resulting from mono-energetic radiation, means pulse height is an unreliable measure for particle classification [2]. It is therefore the pulse-shape features, of certain organic scintillators, that are exploited to distinguish between neutrons and gamma rays.

In addition, the significant tolerances of the gain of photomultiplier tube (PMT) technology means fast neutron assay would benefit from controllable high-voltage (HV) detector supplies integrated with the digital acquisition electronics. A hardware arrangement of this sort allows for gain control with feedback and is necessary for convenient matching of detector responses. Digital analyzers that adopt this arrangement have been recently developed [4] and commercial modules include the CAEN DT5790 [5] and the Hybrid Instruments Ltd. MFAx suite discussed in this paper.

The technology presented was designed specifically to analyze pulse shapes and distinguish events arising from fast scintillators in real time. The current status, more recent developments to the technology and a review of the key measurements made possible with this technology are described. 


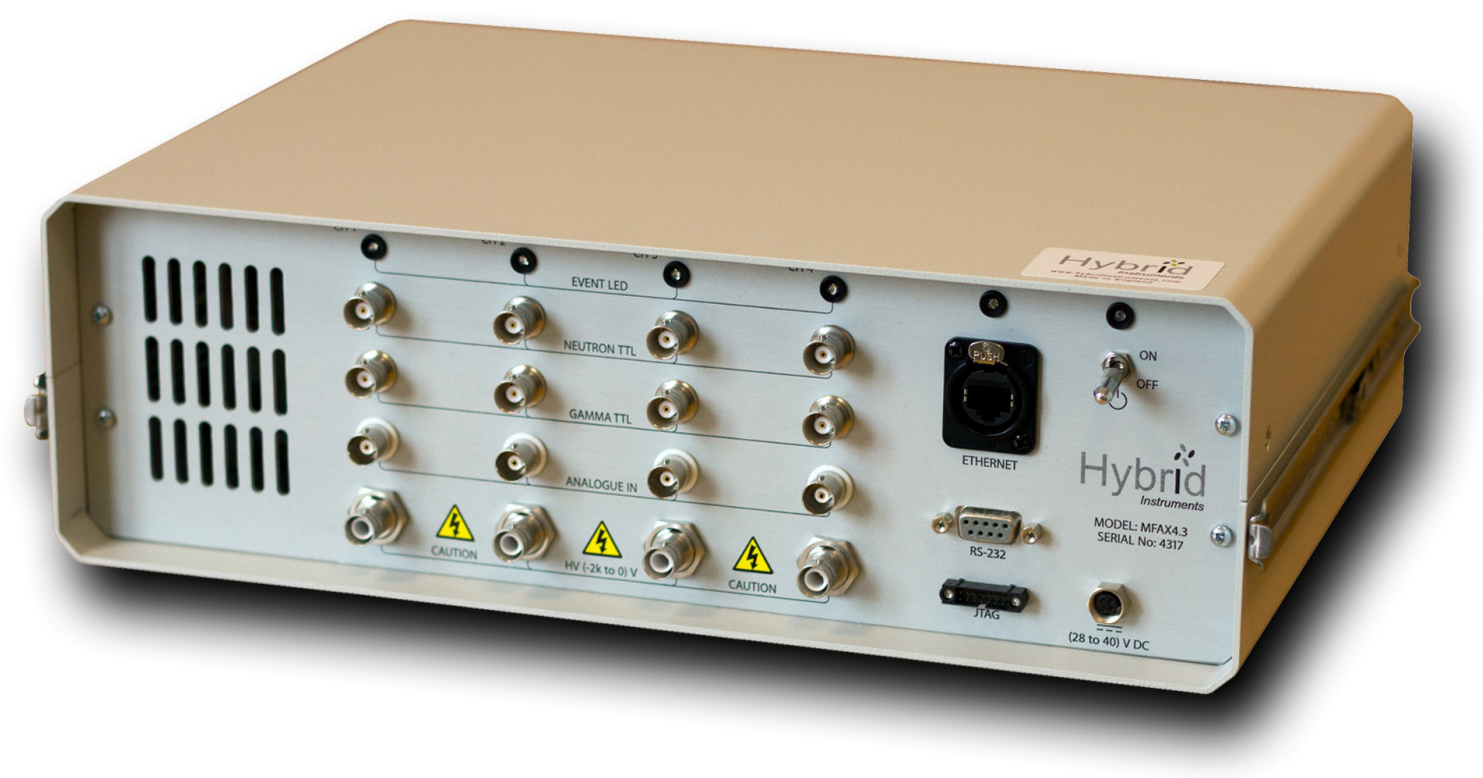

Fig. 1. The front panel of the quad-channel mixed-field analyzer (model no. MFAx4.3). Source: http://www.hybridinstruments.com [6].

\section{Current Status of the Technology}

The Hybrid Instruments Ltd. Mixed-Field Analyzer suite consists of a single channel (MFAx1) and quad-channel (MFAx4) high-speed digitizers designed specifically for realtime Pulse-Shape Discrimination (PSD). These analyzers digitize the analog input signal at 500 million samples per second $(\mathrm{MSa} / \mathrm{s})$ with a 12-bit bipolar Analog-to-Digital Converter (ADC). The following section reports on the current status of the quad-channel digitizer, however, besides the obvious difference in channels available, described functions are consistent across the suite.

Fig. 1 shows the quad-channel MFAx4.3, the point-3 indicating the current version of the technology. All inputs and outputs (IOs) are accessible from the front panel and include analog signal input, HV supply, two Transistor-Transistor Logic (TTL) outputs per channel, Ethernet port, RS-232, power supply, JTAG, activity LED indicators, and power switch. The MFAx4.3 measures $350 \mathrm{~mm} \times 260 \mathrm{~mm} \times 110 \mathrm{~mm}$ and weighs $4.6 \mathrm{~kg}$. Earlier renditions of the technology are reported [7], [8].

The technology is compatible with most legacy fast liquid scintillation detectors (BC510, NE213 and EJ301), low-hazard EJ309 and plastic PSD scintillators (EJ299). Many of the studies summarized here used the fast organic scintillation detectors supplied by Scionix (The Netherlands) based on the EJ309 liquid scintillant (Eljen Technology, TX, USA).

The MFAx offers two output data streams; 1) the TTL digital outputs (two per input channel) for real-time, PSD at rates up to 3 million pulses per second (MPPS) per processing channel, and 2) the Ethernet port for direct PC interface and use of the Graphical User Interface (GUI) software environment. The second method is primarily used for system diagnostics and setup as only a proportion of the PSD data reported to the digital outputs are transmitted over Ethernet, processed and presented by the GUI.
There are two distinguishing features of the MFAx. The first is the ability to perform real-time PSD at high throughput rates of up to 3 MPPS per channel [9]. The event type, determined by PSD, is reported to either neutron or gamma TTL output and are time synchronized with less than $6 \mathrm{~ns}$ jitter to the associated trigger event. The preserved time information of the digital outputs can then be analyzed using proprietary hardware. The Pulse-Gradient Analysis (PGA) [10], [11], [12] PSD algorithm is implemented directly and entirely in VHSIC Hardware Description Language (VHDL) that executes on the FPGA processing core. The second feature, afforded by the close integration of controllable HV supply per analog input channel, is its ability to automatically calibrate detectors' responses by adjusting the supplied HV.

In summary, the true power of this technology is harnessed when the high-speed processing provided by the TTL outputs are utilized. With this arrangement, data at rates of up to 12 MPPS ( $4 \times 3$ MPPS) can be processed by a single MFAx4.3 and therefore, acquisition time can be reduced significantly whilst still maintaining adequate statistics. In some cases, this is the difference between days of acquisition, terabytes of data, and many more hours of post-processing compared to a few hours of measurements and instantaneous results (e.g. [13]).

\section{ReCent Developments to the Technology}

Recent firmware and software developments means that the MFAx hardware can now easily be configured to stream either PSD data or pulse height data via Ethernet to a host PC. When the device is configured to PSD mode 32-bit of data are streamed. These data are constructed of a 2-bit channel identifier, a 2-bit event type, and two 14-bit long and short integral discrimination metrics. When configured to pulseheight mode 32-bit of data are also streamed, consisting of a 2-bit channel identifier, a 2-bit event type, 16-bit unspecified, and a 12-bit baseline-corrected pulse height. The pulse height 


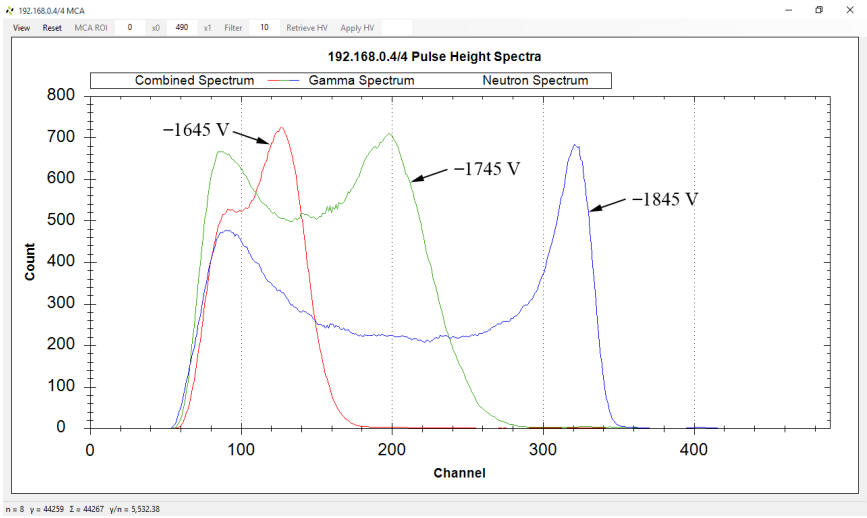

Fig. 2. Cs-137 spectrum with 10-point finite impulse response (FIR) filter acquired from an EJ309 liquid scintillator operated with a negative bias of $1645 \mathrm{~V}, 1745 \mathrm{~V}$ and $1845 \mathrm{~V}$. This illustrates the effect different PMT biases have on the PHS, particularly the location of the Compton edge.

is defined as the maximum single sample amplitude of the unfiltered digitized waveform.

When configured to pulse-height mode the software automatically configures to display Multi-Channel Analyzers (MCA) to report Pulse Height Spectra (PHS). Conveniently, in this mode, PSD is still performed and the event type is attributed to the events data packet. This allows for PHS for combined, gamma-only, and neutron-only data to be obtained. Scintillator-based neutron spectroscopy is challenging due to the random nature by which the neutron energy is deposited in the scintillant, nevertheless neutron spectroscopy afforded by this feature has prompted further investigation into the correlation between neutron pulse height and energy inferred using TOF.

The MCA mode enables the user to quickly and easily adjust HVs to match the responses of multiple detectors based on the PHS. Typically, this would be performed by aligning the response from multiple detectors exposed to a gammaonly source with a defined photopeak (e.g. Cs-137). For a large number of detectors an auto-calibration utility in the software environment has been developed to achieve even faster setup times for experiments where consistency across detector response is essential. The auto-calibration function uses pulse heights acquired in real time to build the Cs-137 PHS, which is used as a measure of the PMT gain. The position of the mode pulse-height in the Compton edge region on the PHS is determined and then the applied HV bias is adjusted to position the mode pulse-height to a user specified MCA channel. This process repeats autonomously until the Compton edges on the PHS are aligned across all detectors. Fig. 2 shows the effect of three different PMT biases on the Cs-137 PHS, and Fig. 3 illustrates the nonlinear relationship between mode pulse-height in the Compton edge region and the supplied PMT bias for one EJ309 detector.

The MCA software interface now offers a real-time embodiment of a finite-impulse-response (FIR) filter. The smoothing effect that this has on the PHS is very beneficial, for both human and computer interpretation, in better determining the MCA channel with the mode pulse-height in the Compton edge region. The magnitude of the filter can be set at any time

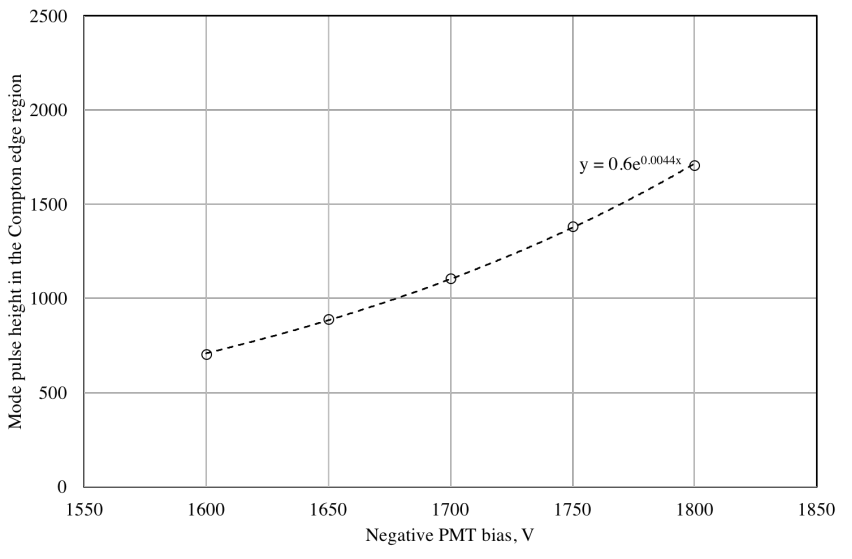

Fig. 3. Nonlinear relationship between mode pulse-height in the Compton edge region and the supplied PMT high-voltage bias. These data were derived from pulse heights acquired from an EJ309 liquid scintillator exposed to a Cs137 source and operated at negative biases ranging from $1600 \mathrm{~V}$ to $1800 \mathrm{~V}$ in $50 \mathrm{~V}$ increments. About 100,000 pulse heights were recorded for each supply bias.

whilst acquiring data, and the filtering influence is displayed in real time. For convenience and to fully exploit the purpose of this function, the user is now also able to control the detectors HV directly from the MCA interface. This provides an indication of the result of a newly applied HV on the PHS within minutes (typically the time taken for the HV to stabilize and acquire an adequate data set). Fig. 4 and Fig. 5 show an unfiltered and 20-point filtered gamma-only PHS of Cs137 data acquired from an EJ309 liquid scintillator. Fig. 6 shows combined, gamma-only and neutron-only PHS for Cf252 , filtered with a 20-point moving average and acquired with the same configured hardware used to obtain Fig. 4 and Fig. 5. The menu bar at the top of the MCA interface clearly displays options to focus on a region-of-interest (ROI), apply a filter, retrieve the current $\mathrm{HV}$, and apply a new HV setting.

One of the most significant recent advances is the shift from all configurable settings being stored only in the hardware's memory to also having a replica of settings stored in an .INI configuration file, which typically resides on the host PC.

Previously when the software was executed it sent a command to the hardware requesting all settings to be downloaded to the PC-based software where the user could then view, edit and upload revised settings. This design requires no synchronization of variables, but requires the user to carefully document the settings as no history is kept. Furthermore, it limits the ease to quickly set-up other hardware with the same settings. Hardware can operate independently from the host $\mathrm{PC}$, even from a power cycle because settings are uploaded from the hardware memory during the boot sequence.

With the new design, configuration files are automatically saved and dated and can be quickly transferred to another PC and uploaded to another hardware device. However, synchronization of settings becomes an issue using this method. The challenge is in knowing if the settings viewed in the software environment obtained from the configuration file are identical to the settings stored in the memory of the hardware. This issue is addressed by a more comprehensive initialization procedure 


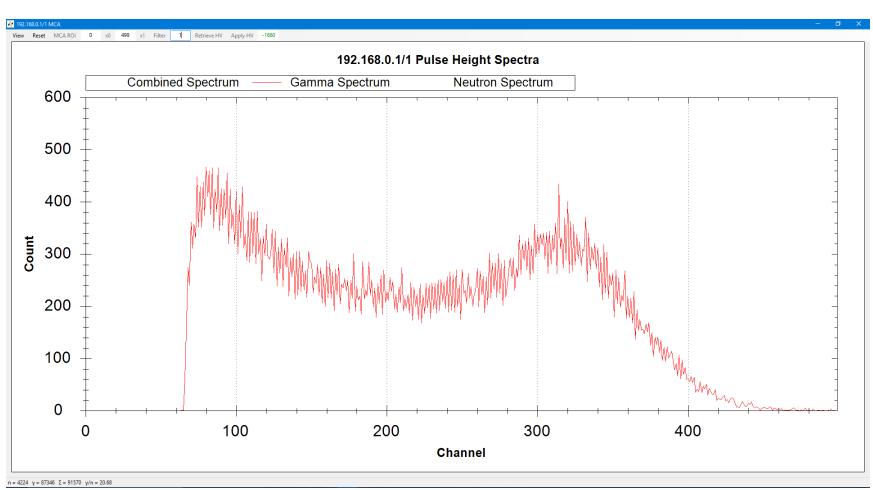

Fig. 4. The MCA graphical-interface with unfiltered gamma-only PHS of data acquired from an EJ309 liquid scintillator positioned $45 \mathrm{~mm}$ from a Cs-137 source.

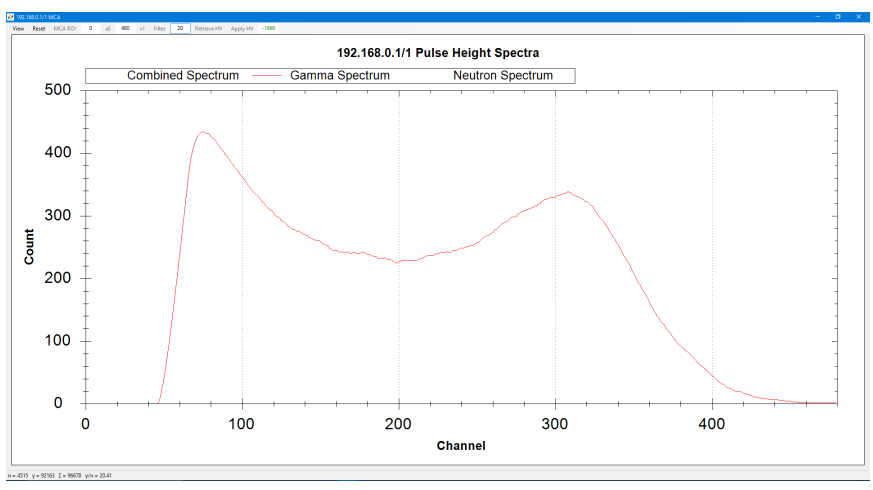

Fig. 5. The MCA graphical-interface with 20-point moving-average filtered gamma-only PHS of data acquired from an EJ309 liquid scintillator positioned $45 \mathrm{~mm}$ from a Cs-137 source.

performed by the software and is described in detail below.

An Ethernet cable and PC can be connected to the hardware at any time during its operation. When the software is executed PC ARP requests are sent asynchronously to all potential IP addresses. Once the hardware responds with an ARP reply, the software is aware of what MFAs are online. Using transmission control protocol (TCP) the PC sends a request command for all settings to be downloaded from all MFAx hardware connected. Once the PC-based software has received all hardware settings the configuration file loads from wherever it resides on the PC. If a conflict exists between the detected hardware and the expected hardware, as defined by the configuration file, then the user is informed and presented with four options; 1) Rescan for hardware changes, 2) Target alternative configuration file, 3) Create new configuration file based on current hardware settings, or 4) Exit application. Following this, the settings retrieved from the configuration file are compared to the settings downloaded from the hardware. If discrepancies exist between the settings the user is informed and presented with four options; 1) Upload new configuration file settings to hardware, 2) Target alternative configuration file, 3) Create new configuration file based on current hardware settings, or 4) Exit application. The software is fully compatible with any number of connected MFAx devices from the suite, and will automatically adapt to represent the hardware that is registered as online.

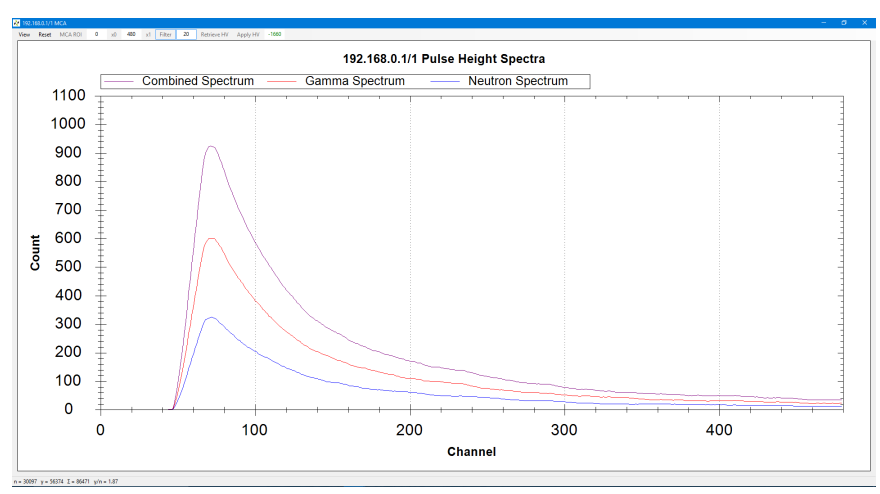

Fig. 6. The MCA graphical-interface with 20-point moving-average filtered combined, gamma-only and neutron-only PHS of data acquired from an EJ309 liquid scintillator positioned on axis from a water-moderated Cf-252 source.

\section{A Review of the Measurements}

Many measurements have been performed and reported that use the MFAx and include work by the IAEA, the Joint Research Centre (JRC), Pajarito Scientific Corporation (PSC), the National Physical Laboratory (NPL) and Lancaster University. Specific details, including references, of these measurement campaigns are given in the following paragraphs. In most of these reports the users have exploited the real-time, PSD throughput capability of 3 MPPS per channel via the TTL data streams and the integrated detector HV supplies, whilst also benefiting from many of the other inherent features supported by the hardware and software environment.

Suggestions and examples of successfully implemented proprietary hardware for the analysis of the TTL outputs are; 1) a National Instruments Industrial Controller. The controller includes an FPGA-based data acquisition card and custom-built analysis software developed in LabVIEW [7], [14]. 2) custommade de-randomizing electronics (Los Alamos National Laboratory, NM) connected to a JSR-15 multiplicity shift register (Canberra Industries Inc., CT) for the measurement of Uranium-235 enrichment in small samples of low-enriched triuranium octoxide [15], and 3) a bespoke 32-bit, 64-channel, binary counter with a serial PC interface (Lancaster University, UK) for reactor imaging [13] and neutron tomography using an isotropic source [16]. In addition, the commercially available multichannel Pulse Train Recorder (model: PTR-32) by the Institute of Isotopes (Hungarian Academy of Sciences or Energia, Budapest) is also fully compatible with the MFAx technology and offers an embedded de-randomizing function.

The technology has been used with a fast, organic liquid scintillator for imaging an operational TRIGA mark II nuclear reactor [13]. This study utilized a lightweight and portable system comprising of a single channel MFA, detector, collimator and equatorial mount [17], [18]. This compact singledetector system overcomes the problems of other imagers reported in the literature that are not portable and use large arrays of detectors [19], [20], and are therefore not easily transferable to areas with limited physical access. The data acquired during this campaign comprised of a matrix with 9504 elements corresponding to different orientations of the detector and collimator. Sufficient data were acquired at each 
point in $500 \mathrm{~ms}$; made possible by the MFAs ability to perform real-time PSD at rates up to 3 MPPS.

Developed in collaboration between the IAEA, JRC and Hybrid Instruments Ltd., the MFA has been implemented in a Liquid Scintillator Neutron Coincidence Collar (LS-NCC) as an alternative to the ${ }^{3} \mathrm{He}$-based Uranium Neutron Collar (UNCL) and showed potential for better performance [21]. The fast-neutron detection capabilities of the LS-NCC are particularly beneficial for coincidence counting in safeguards applications. Detection of neutrons from fission events does not require a thermalization process, thus all neutrons coming from the same event are detected with almost zero delay; event detection made possible by the parallel processing and extremely low dead-time of the MFA technology. This feature makes it possible to reduce the detector coincidence gate resulting in significantly lower statistical measurement error. Furthermore, the autocalibration function allows for the twelve detectors used in the LS-NCC to be response matched reliably, quickly and easily by an operator requiring speed and repeatability, such as a field analyst.

Other studies utilizing fast-neutron multiplicity and coincidence counting with a diverse range of potential applications including nuclear material accountancy in storage, criticality assurance, non-proliferation, nuclear safeguards and security have also benefited from the capabilities of this technology [7], [9], [14], [22], [23], [24], [25], [26], [27].

\section{CONCLUSIONS}

This paper has reviewed the recent firmware and software advances made to the MFA technology developed by Hybrid Instruments Ltd. These advances include the addition of an MCA mode (including filter) for displaying PHS spectra, an automatic utility for reliable detector response-matching and a new process for conveniently porting hardware configurations from one device to another using a configuration file that resides on the host PC. The underlying strength of this technology comes from its ability to perform real-time PSD and report these data on dedicated digital outputs at rates of up to 3 MPPS per channel.

The MFA technology has supported many real-time measurement campaigns, has been used with four different EJ309 detector geometries [14] and is compatible with several multiplicity analyzers. Some of the most novel measurements, made possible using this technology, are reviewed.

Technologies of this sort are constantly improving with greater sampling frequency, amplitude bit resolution, number of channels, lower dead-time and higher throughput. Efforts are also being made to reduce power consumption and form factor to increase portability and remove the reliance on mains power. Of the many different devices available on the market, the 'go-to' device based on attributes such as sampling frequency, bit resolution, channels available, data rate, data recorded and other non-specific features, depend very much on the individual needs of that particular measurement campaign. The device described in this work is capable of processing large volumes of events in relatively short acquisition times from multiple detectors with real-time analysis and so is particularly suited to the study of events with low detection probability such as neutron coincidence counting double, triple and quad events.

\section{ACKNOWLEDGMENT}

We thank all users of the MFAx technology, particularly the researchers at Lancaster University, the IAEA and Alice Tomanin at the JRC. We acknowledge the support offered during early stage testing and for providing essential feedback and useful discussions during the development of the technology described. We acknowledge the support of the EPSRC via grant EP/L025671/1 and M. J. Joyce acknowledges the receipt of a Royal Society Wolfson Research Merit award.

\section{REFERENCES}

[1] R. Jebalia et al., "A first comparison of the responses of a 4He-based fast-neutron detector and a NE-213 liquid-scintillator reference detector," Nucl. Instruments and Methods in Physics Res. A, vol. 794, pp. 102-108, Sep. 2015.

[2] T. W. Crane, M. P. Baker, D. Reilly, N. Ensslin, and H. Smith, Passive Nondestructive Assay of Nuclear Materials. Los Alamos National Laboratory, Mar. 1991, vol. LA-UR-90-732.

[3] D. C. Stromswold, A. J. Peurrung, R. R. Hansen, and P. L. Reeder, "Direct fast-neutron detection," Pacific Northwest National Laboratory, Richland, Washington 99352, Tech. Rep. PNNL-13068, 1999.

[4] A. A. Ivanova et al., "Fast neutron flux analyzer with real-time digital pulse shape discrimination," Nucl. Instruments and Methods in Physics Res. A, vol. 827, no. 4, pp. 13-17, August 2016.

[5] CAEN. (2016, Oct.) DT5790, Digital Pulse Analyzer. [Online]. Available: http://www.caen.it/csite/CaenProd.jsp?idmod=896\&parent=65\#

[6] Hybrid Instruments Ltd. (2016, Oct.). [Online]. Available: http://www.hybridinstruments.com/index.html

[7] M. J. Joyce, K. A. A. Gamage, M. D. Aspinall, F. D. Cave, and A. D. Lavietes, "Real-time, fast neutron coincidence assay of plutonium with a 4-channel multiplexed analyzer and organic scintillators," IEEE Trans. Nucl. Sci., vol. 61, no. 3, pp. 1340-1348, Jun. 2014.

[8] M. J. Joyce, M. D. Aspinall, F. D. Cave, K. Georgopoulos, and Z. Jarrah, "The design, build and test of a digital analyzer for mixed radiation fields," in IEEE 1st Int. Conf. on Advancements in Nucl. Instrum. Meas. Methods and their Appl. (ANIMMA), Marseille, France, 2009.

[9] M. J. Joyce, M. D. Aspinall, F. D. Cave, and A. D. Lavietes, "Realtime, digital pulse-shape discrimination in non-hazardous fast liquid scintillation detectors: Prospects for safety and security," IEEE Trans. Nucl. Sci., vol. 59, no. 4, pp. 1245-1251, Aug. 2012.

[10] M. J. Joyce, K. A. A. Gamage, M. D. Aspinall, F. D. Cave, and A. D. Lavietes, "A 4-channel multiplex analyzer for real-time, parallel processing of fast scintillators," in IEEE Nucl. Sci. Symp. and Med. Imag. Conf. (NSS/MIC), Anaheim, CA, 2012.

[11] B. D'Mellow, M. D. Aspinall, R. O. Mackin, M. J. Joyce, and A. J. Peyton, "Digital discrimination of neutrons and $\gamma$-rays in liquid scintillators using pulse gradient analysis," Nucl. Instruments and Methods in Physics Res. A, vol. 578, no. 1, pp. 191-197, Jul. 2007.

[12] M. D. Aspinall et al., "Verification of the digital discrimination of neutrons and gamma rays using pulse gradient analysis by digital measurement of time-of-flight," Nucl. Instruments and Methods in Physics Res. A, vol. 583, pp. 432-438, 2007.

[13] J. S. Beaumont, M. P. Mellor, M. Villa, and M. J. Joyce, "High-intensity power-resolved radiation imaging of an operational nuclear reactor," Nature Communications, vol. 6, p. 8592, Oct. 2015.

[14] A. D. Lavietes et al., "Liquid scintillator-based neutron detector development," in IEEE Nucl. Sci. Symp. and Med. Imag. Conf. (NSS/MIC), Anaheim, CA, 2012, pp. 230-244.

[15] H. M. O. Parker et al., "Active fast neutron singles assay of $235 \mathrm{U}$ enrichment in small samples of triuranium octoxide," Progress in Nucl. Energy., vol. 93, pp. 59-66, 2016.

[16] M. J. Joyce, S. Agar, M. D. Aspinall, J. S. Beaumont, E. Colley, M. Colling, J. Dykes, P. Kardasopoulos, and K. Mitton, "Fast neutron tomography with real-time pulse-shape discrimination in organic scintillation detectors," Nucl. Instruments and Methods in Physics Res. A, vol. 834, pp. 36-45, Oct. 2016. 
[17] K. A. A. Gamage, M. J. Joyce, and G. C. Taylor, "A digital approach to neutron-gamma imaging with a narrow tungsten collimator aperture and a fast organic liquid scintillator detector," Applied Radiation and Isotopes, vol. 70, pp. 1223-1227, 2012.

[18] K. A. A. Gamage, M. J. Joyce, and J. C. Adams, "Combined digital imaging of mixed-field radioactivity with a single detector," Nucl. Instruments and Methods in Physics Res. A, vol. 635, pp. 74-77, 2011.

[19] A. Poitrasson-Rivière et al., "Dual-particle imaging system based on simultaneous detection of photon and neutron collision events," Nucl. Instruments and Methods in Physics Res. A, vol. 760, pp. 40-45, Oct. 2011.

[20] N. Mascarenhas, J. Brennan, K. Krenz, P. Marleau, and S. Mrowka, "Results with the neutron scatter camera," IEEE Trans. Nucl. Sci., vol. 56, no. 3, pp. 1269-1273, Jun. 2009.

[21] D. Henzlova et al., "Current status of helium-3 alternative technologies for nuclear safeguards," in National Nuclear Security Administration, U.S. Department of Energy, and The Euratom Atomic Energy Community (Euratom), Ispra, Italy, 2015.

[22] R. Sarwar, M. J. Joyce, and C. H. Zimmerman, "Real-time fast-neutron plutonium assay for storage and ageing applications," in 2nd Annual Meeting DISTINCTIVE University Consortium, Bristol, UK, 2016.

[23] M. J. Joyce, J. Adamczyk, M. D. Aspinall, F. D. Cave, and R. Plenteda "Real-time, fast neutron detection for stimulated safeguards assay," in Proceedings of 37th ESARDA Symp. on Safeguards and Nucl. NonProliferation, Manchester, UK, 2015.

[24] M. J. Joyce, M. D. Aspinall, F. D. Cave, and A. D. Lavietes, "A 16channel real-time digital processor for pulse-shape discrimination in multiplicity assay," IEEE Trans. Nucl. Sci., vol. 61, no. 4, pp. 22222227, Aug. 2014.

[25] M. J. Joyce, "Fast neutron multiplicity assay," Nucl. Future, vol. 9, no. 6 , pp. 43-46, Nov. 2013.

[26] A. D. Lavietes et al., "Real-time measurements of production uranium fuel assemblies using a liquid scintillator-based active interrogation neutron detection system," in IEEE Nucl. Sci. Symp. and Med. Imag. Conf. (NSS/MIC), Seoul, South Korea, 2013.

[27] _ , "Development of a liquid scintillator-based active interrogation system for LEU fuel assemblies," in IEEE 1st Int. Conf. on Advancements in Nucl. Instrum. Meas. Methods and their Appl. (ANIMMA), Marseille, France, 2013. 